\title{
I-Q imbalance correction in time and frequency domains with application to pulse doppler radar
}

\author{
N SIVANNARAYANA and K VEERABHADRA RAO \\ SHL, Research Centre Imarat, Vignyanakancha, Hyderabad 69, India \\ e-mail: siva@rci.ernet.in
}

\begin{abstract}
Digital In-phase(I) and Quadrature phase(Q) imbalance correction schemes are presented for improving the balance between I \& $Q$ signals by rejecting the image frequencies due to imbalance. The imbalance errors in the analog and digital demodulation schemes are highlighted. Simplified correction schemes are presented for time and frequency domain imbalances. These correction schemes are useful in radar and communication systems. In this paper, a digital I-Q scheme is also presented for a pulse Doppler radar, along with hardware configuration for implementation.
\end{abstract}

Keywords. Digital I-Q; quadrature demodulation; pulse doppler radar; timefrequency analysis.

\section{Introduction}

In a coherent receiver, In-phase and Quadrature (I-Q) phase signals are derived by demodulating the Intermediate Frequency (IF) signal. The I-Q signals should match in gain and in phase by 90 degrees. In the analog schemes, IF signal is demodulated using in-phase and quadrature phase carrier (Goldman 1986; Liu 1989; Tsui 1995) and is sampled in two channels. In the digital schemes (Liu et al 1989, Tsui). IF signal is sampled and demodulated using sampled cosine and sine of the carrier. These I-Q schemes are explained in the literature towards simplification in the implementation (Levanon 1988; Liu et al 1989). However, these schemes tend to develop amplitude and phase imbalances. In this work, we are proposing simplified schemes for correcting the imbalances in time and frequency domains with application to pulse Doppler radar.

In radar and communication systems, signals are sampled for digital processing. As per Nyquist criterion a signal must be sampled at a rate more than twice the bandwidth. In complex (I-Q) sampling, the signals can be sampled minimum at the rate of bandwidth. I-Q signals avoid blind phases in sampling. This complex sampling also improves signal to noise ratio (SNR) by $3-\mathrm{dB}$ compared to the only real (I-channel) signal processing (Levanon 1988). 
The analog I-Q detector has a limitation of matching the gain \& phase in the I and Q channels which can be achieved only upto a certain level (Goldman 1986). The mismatch creates an unwanted image frequency with an amplitude $24 \mathrm{~dB}$ down to the main signal. To achieve further rejection, the penalty in terms of cost is very high (Goldman 1986). Correction algorithms are incorporated in the signal processors to reduce the imbalance.

In the literature (Liu et al 1989; Tsui 1995), digital I-Q schemes are presented. Analog I-Q imbalance corrections are explained by Churchill et al (1981) and Levanon (1988) and the calibration procedure is presented by Pierre \& Fuhrmann (1995). Hilbert transform techniques are described by Oppenheim \& Schafer (1975). Simplification of digital sampling is available by Brown (1979), Considine (1983), and Frerking (1994). Recent literature (Liu et al 1989; Tsui 1995) on I-Q, stresses the imbalance free demodulation. However, such demodulation creates an additional imbalance due to delay between I-Q samples for Doppler-shifted signals. For example, in radars, the image frequencies have to be rejected up to $50 \mathrm{~dB}$ to $60 \mathrm{~dB}$ down in severe clutter to signal levels of 30 to $40 \mathrm{~dB}$. From the clutter signal, the image frequencies can be calculated, but ignoring of these image frequencies creates additional blind zones in the Doppler plane. So to avoid blind zones and false target detections, the image frequency level should be brought down to the noise background.

\section{Modeling I-Q imbalances}

I-Q channels are modeled in four ways depending on the presence of error terms either in I or Q channels. The most commonly used signal with errors is (Churchill et al 1981; Levanon 1988)

$$
x(t)=a(t)[(1+\varepsilon) \cos (\omega t)+j \sin (\omega t+\theta)],
$$

where $a(t)$ is the envelope, ' $\omega$ ' is the angular frequency, ' $\varepsilon$ ' is the amplitude imbalance, ' $\theta$ ' is the phase imbalance between I-Q channels and ' $t$ ' is the time. The imbalance ratio is derived for (1). For simplicity $a(t)$ is taken as amplitude $A$.

$$
\begin{aligned}
x(t) & =A[(1+\varepsilon) \cos (\omega t)+j \sin (\omega t+\theta)] \\
& =A / 2\left[(1+\varepsilon)\left(e^{j w t}+e^{-j w t}\right)+\left(e^{j(w t+\theta)}-e^{-j(w t+\theta)}\right)\right] \\
& =A / 2\left[e^{j u t}\left(1+\varepsilon+e^{j \theta}\right)+e^{-j w t}\left(1+\varepsilon-e^{-j \theta}\right)\right] .
\end{aligned}
$$

The image frequency strength $X(-\omega)$ to main component strength $X(\omega)$ is given by

$$
\begin{aligned}
\frac{X(-\omega)}{X(\omega)} & =\frac{\left(1+\varepsilon-e^{-j \theta}\right)}{\left(1+\varepsilon+e^{j \theta}\right)} \\
& =\frac{[1+\varepsilon-\cos (\theta)+j \sin (\theta)]}{[1+\varepsilon+\cos (\theta)+j \sin (\theta)]} \\
& =\frac{\left[\varepsilon^{2}+2 \varepsilon+j 2(1+\varepsilon) \sin (\theta)\right]}{\left[2\left(1+\varepsilon+\varepsilon^{2} / 2+\varepsilon \cos (\theta)+\cos (\theta)\right)\right]} \\
& \approx \varepsilon / 2+j \theta / 2, \text { for small values of } \varepsilon \text { and } \theta .
\end{aligned}
$$


Power ratio $P_{-\omega} / P_{\omega}$ is derived by taking $|X(-\omega)|^{2} /|X(\omega)|^{2}$ and is given by (Churchill et al 1981; Levanon 1988; Liu et al 1989)

$$
P_{-\omega} / P_{\omega} \approx\left(\varepsilon^{2}+\theta^{2}\right) / 4
$$

Similarly we can derive expressions for the remaining three cases. For the four possible imbalance cases, the image to main signal strength is given by

$$
\begin{array}{ll}
\text { Signal model } x(t)= & X(-\omega) / X(\omega) \approx \\
A[(1+\varepsilon) \cos (\omega t)+j \sin (\omega t+\theta)], & (\varepsilon / 2+j \theta / 2), \\
A[\cos (\omega t)+j(1+\varepsilon) \sin (\omega t+\theta)], & (-\varepsilon / 2+j \theta / 2), \\
A[\cos (\omega t+\theta)+j(1+\varepsilon) \sin (\omega t)], & (-\varepsilon / 2-j \theta / 2), \\
A[(1+\varepsilon) \cos (\omega t+\theta)+j \sin (\omega t)], & (\varepsilon / 2-j \theta / 2),
\end{array}
$$

Imbalance errors can be estimated and corrected using frequency domain data while performing calibration. In order to calibrate, reference input is given at different frequencies preferably covering the whole band. Depending on the selected model, the ratio $X(-\omega) / X(\omega)$ is considered with proper polarity. This is useful in automated calibration of receivers (Pierre \& Fuhrmann 1995).

\section{Imbalance correction to the analog I-Q demodulators}

In the analog I-Q, imbalances exist while demodulating the input using sine and cosine carriers at intermediate frequency. Imbalance correction for analog I-Q detector is presented below.

\subsection{Imbalance correction in the time domain}

The I-Q signals with imbalances of case-1, (2a) is modeled as

$$
\begin{aligned}
& I(t)=A(1+\varepsilon) \cos (\omega t), \\
& Q(t)=A \sin (\omega t+\theta) .
\end{aligned}
$$

This can be corrected digitally by transforming the data as (Levanon 1988)

$$
\begin{aligned}
I 1(t) & =E I(t), \\
Q 1(t) & =P I(t)+Q(t),
\end{aligned}
$$

where $E=\cos (\theta) /(1+\varepsilon)$ and $P=-\sin (\theta) /(1+\varepsilon)$.

After transformation,

$$
\begin{aligned}
& I 1(t)=A \cos (\theta) \cos (\omega t), \\
& Q 1(t)=A \cos (\theta) \sin (\omega t) .
\end{aligned}
$$

The scheme mentioned above requires correction both in $I \& Q$ channel paths. Alternatively, we propose to correct this imbalance in the Q-channel path only by 
modifying (4). This modified scheme is suitable for easy implementation in the signal processors.

$$
\begin{aligned}
I 1(t) & =I(t) \quad(\text { remains same as input }) \\
Q 1(t) & =(P / E) I(t)+(1 / E) Q(t)
\end{aligned}
$$

\subsection{Imbalance correction in the frequency domain}

In this scheme, analog baseband I-Q signals are sampled and the error correction is applied in the frequency domain. Normally, frequency transformation like discrete fourier transform (DFT) will be performed on the sampled data. The image frequency component appears after transformation. Hence, the correction is applied uniformly for all the bins. In the frequency domain, the frequency bins beyond $f_{s} / 2$ are complex-conjugates of the bins upto $f_{s} / 2$ and vice versa (Oppenheim \& Schafer 1975) ( $f_{s}$ is the sampling frequency). Hence, both amplitude scaling and phase rotation, i.e. $(\varepsilon / 2+j \theta / 2)$ is provided uniformly on all the bins after conjugation operation.

For $N$-point DFT, let $X(k)$ denote the transformed output and $X^{*}(k)$ its conjugate; index $k$ goes from 0 to $N-1$. The correction data $X M$ is generated as

$$
X M(k)=(\varepsilon / 2+j \theta / 2) X^{*}(k) ; \quad k=1,2, \ldots, N-1,
$$

and the corrected data $\mathrm{XC}(\mathrm{k})$ is given by

$$
X C(k)=X(k)-X M(N-k) ; \quad k=1,2, \ldots, N-1 .
$$

$X C(0)=X(0)$ in all $X C$ computation equations.

With this type of correction, the image frequency component strength can be reduced by 30 to $40 \mathrm{~dB}$ below the main component strength.

\section{Digital I-Q generation}

In a digital I-Q scheme, the carrier (IF) is offset at half the bandwidth B/2 (Brown 1979; Considine 1983; Goldman 1986; Tsui 1995) and is sampled at $2 \mathrm{~B}$ frequency. Demodulation is performed using $\cos (n \pi / 2) \& \sin (n \pi / 2)$ which takes $\{-1,0,1\}$ for integer values of ' $n$ '. Thus, demodulation is a simple operation of changing the polarity of the input depending on $\{-1,1\}$. In this scheme, $\cos (n \pi / 2)$ is ' 1 ' when $\sin (n \pi / 2)$ is ' 0 ' and vice versa. So, Qchannel is a delayed version by a sampling interval. This delay creates an image frequency component. A fixed delay creates linear phase shift with frequency (Oppenheim \& Schafer 1975). In analog I-Q scheme, phase shift is constant.

\subsection{Imbalance correction in the frequency domain for digital $I-Q$ scheme}

The sampled signals when analyzed in the frequency domain, give linearly varying phase shift with bin number. Hence, every correction scheme requires the frequency bin dependent phase correction. For a fixed sampling clock, correction coefficients are generated as function of the bin number. 
Let $f_{s}$ be the sampling frequency for I-Q signals and delay between I-Q samples is $\delta t$. Sampling interval $t_{s}$ and $\delta t$ need not be related. Normally, $\delta t$ is less than $t_{s}$. In pulse Doppler radars $t_{s}$ is the pulse repetitive interval and $\delta t$ is equal to $1 / 2 \mathrm{~B}$ for the signal sampled at $2 \mathrm{~B}$.

$$
\begin{aligned}
x(t) & =A[\cos (\omega t)+j \sin (\omega t+\omega \delta t)] \\
& =A / 2\left[\left(e^{j \omega t}+e^{-j \omega t}\right)+\left(e^{j(\omega t+\omega \delta t)}-e^{-j(\omega t+\omega \delta t)}\right)\right] \\
& =A / 2\left[e^{j \omega t}\left(1+e^{j \omega \delta t}\right)+e^{-j \omega t}\left(1-e^{-j \omega \delta t}\right)\right] .
\end{aligned}
$$

The image strength $X(-\omega)$ to main component strength $X(\omega)$ is given by

$$
\begin{aligned}
X(-\omega) / X(\omega) & =\left(1-e^{-j \omega \delta t}\right) /\left(1+e^{j \omega \delta t}\right) \\
& =j[\sin (\omega \delta t) /(1+\cos (\omega \delta t))] \\
& \approx j \omega \delta t / 2
\end{aligned}
$$

' $\omega \delta t^{\prime}$ ' is denoted as $\Phi(k)$ in the discrete frequency domain. Phase error for a bin of one (i.e. $f_{s} / N$ ) of $N$-point DFT is given by

$$
\Phi_{b}=2 \pi\left(f_{s} / N\right) \delta t
$$

Phase error for other bins is given by

$$
\begin{aligned}
\Phi(k) & =\Phi_{b} k ; \quad k=0,1, \ldots, N-1 \\
X M(k) & =j[\sin (\Phi(k)) /(1+\cos (\Phi(k)))] X^{*}(k) \\
& \approx j[\Phi(k) / 2] X^{*}(k) .
\end{aligned}
$$

Equation (11) does not impose any constraint, and (12) limits the rejection for higher delays $(\delta t)$ between I-Q signals. Image-free components $X C(k)$ are generated using $(7)$.

Windowing is applied on the time domain data to reduce the sidelobes. Figure 1a shows the input spectrum and figure $1 \mathrm{~b}$ is the $X M(k)$. Figure $1 \mathrm{c}$ is the frequency reversal of $X M$ and this is subtracted from input and indicated in figure 1d, which indicates the imagecorrected spectrum. The main component is at bin 60 and image is at bin 4 . Blackman window is used for this.

Due to windowing, frequency spread increases (Levanon 1988; Oppenheim \& Schafer 1975 ). Let $N-2$ be the excitation bin and due to frequency domain broadening, $N-3$ $\& N-1$ bins are also excited. For all these three bins, phase shift is $(N-2) \Phi_{b}$. For $(N-1)$ th bin, phase correction weight is $(N-2) \Phi_{b}$. But, the correction applied is given as $(N-1) \Phi_{b}$ in (12). The error rejection is $((N-1)-(N-2)) /(N-2) \approx(1 / N)$, hence, at higher bin numbers, the rejection improvement is limited to $20 \log (N)$.

For example, using a 64-point FFT, the possible rejection is around $20 \log (64)=36 \mathrm{~dB}$. Prior to correction, the image frequency ratio is of the order of $-14 \mathrm{~dB}$ for the bin number 60 (image bin at 4 ) and $-18 \mathrm{~dB}$ image for bin number 61 (image bin at 3) as shown in figure 1a. After correction, the image is reduced by approximately $36 \mathrm{~dB}$. With this technique, the image to main component strength is $-54 \mathrm{~dB}$ as shown in figure $1 \mathrm{~d}$. All the graphs are normalized with respect to the input spectrum maxima. Smaller values of the spectrum are limited to $-80 \mathrm{~dB}$ in displaying figure 1 . 

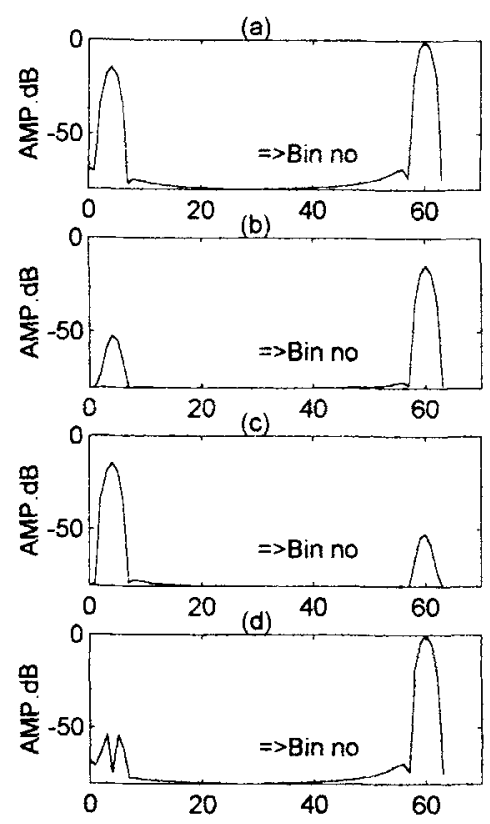

Figure 1. Frequency domain correction - magnitude plots. Phase is not indicated in the plots. (a) Input signal spectrum, $X(k)$, main component at bin $60 \&$ its image at bin 4 . (b) Correction spectrum, $X M(k)$, this takes care of phase also. (c) Frequency reversed of $X M(k)$. (d) Corrected spectrum. This is generated by subtracting $X M(-k)$ from $X(k)$.

\subsection{Imbalance correction in the time domain for the modified digital scheme}

The time delay between I-Q signals requires frequency dependent correction. As explained in the previous section in the frequency domain, correction vector is generated as frequency reversed conjugated spectrum with bin dependent coefficients. Thus, this forms multiplication in the frequency domain. Multiplication in the frequency domain reflects as convolution in the time domain. Convolution operation on the input gives correction signal in the time domain.

The corrected spectrum is given by,

$$
\begin{aligned}
X C(k) & =X(k)-X M(N-k) ; \quad k=1,2, \ldots, N-1 . \\
\text { Let } H(k) & =j[\sin (\Phi(k)) /(1+\cos (\Phi(k)))], \\
\text { and } h(n) & =I D F T[H(k)],
\end{aligned}
$$

where IDFT denotes the inverse DFT operation. The number of significant coefficients in $h(n)$ are much less compared to $N$. The image free signal $x_{C}(n)$ is generated as,

$$
\begin{aligned}
x c(n) & =x(n)-x m(n), \\
x m(n) & =h(-n) \odot x^{*}(n),
\end{aligned}
$$

where $\odot$ denotes circular convolution. Circular convolution is a computationally involved operation compared to the frequency domain correction. Also, time domain correction scheme lacks the facility for auto-calibration of the receiver.

While utilizing this scheme the following observations are to be noted

(a) Filter consists of only few significant coefficients. Hence, the filter coefficients can be truncated to 5 to 8 coefficients, which saves computation time. 


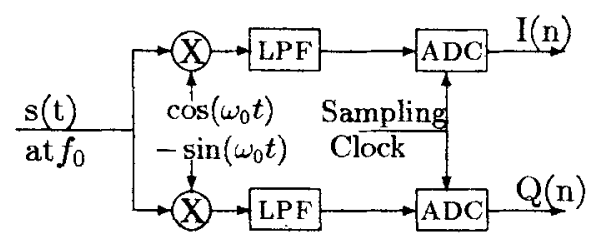

Figure 2. Analog I-Q scheme.

(b) Circular convolution is performed on the finite data block.

(c) The number of filter weights along with FFT size determine the possible imbalance reduction.

\section{I-Q scheme for pulse doppler radar}

In pulse Doppler radars, carrier at transmitted frequency is pulse modulated and received echo is first converted to the intermediate frequency(IF). While converting the signal from IF to baseband, I-Q signals are generated as indicated in figure 2. In this scheme, carrier at IF is demodulated using $e^{-j w_{0} t}$ and the output is filtered using a low-pass filter (LPF) of bandwidth approximately equal to the reciprocal of the pulse width. I-Q signals are sampled using two Analog to Digital Converters (ADCs) at sampling interval less than or equal to the transmitted pulse width $(\tau)$.

Digital equivalent of the scheme is indicated in figure 3 . In this scheme IF is created at $f_{0}(=1 / \tau)$ and sampled at $4 f_{0}$. This signal is multiplied with a carrier $\exp \left[-j\left(2 \pi f_{0} n\left(1 / 4 f_{0}\right)\right)\right]=\cos (n \pi / 2)-j \sin (n \pi / 2)$ and is suitably low-pass filtered and down sampled to generate the required output sampling rate.

As an example, transmitter pulse width of $400 \mathrm{~ns}$. is considered. The Steps involved here are as below.

(a) Create IF $\left(f_{0}\right)$ at $2.5 \mathrm{MHz}$, one cycle is created in $400 \mathrm{~ns}$. width.

(b) Sample at $10 \mathrm{MHz}\left(4 f_{0}\right)$.

(c) Generate $\cos (n \pi / 2)\left[\begin{array}{lllllllll}1 & 0 & -1 & 0 & 1 & 0 & -1 & 0 & \ldots\end{array}\right]$ period of 4 samples.

(d) Generate $\sin (n \pi / 2)\left[\begin{array}{llllllll}0 & 1 & 0 & -1 & 0 & 1 & 0 & -1\end{array}\right]$ period of 4 samples.

(e) Pass through LPF to pass signals up to $f_{0}$ and remove components greater than $2 f_{0}$. The filter should have maximum of 4 taps (equal to pulse width).

(f) Decimate the filtered output by 2 to 4 , to generate the I-Q signals at the required sampling frequency.

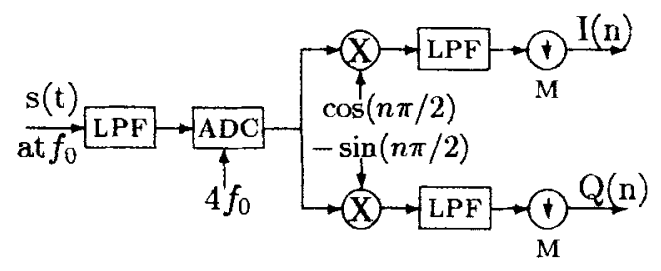

Figure 3. Digital I-Q scheme. 


\subsection{Choice of filter weights}

Transmitted pulse is contained in approximately 4 samples. Matched filtering keeps the filter with samples comparable to the input. Filtering extends the signal due to convolution. Hence, the filter taps have to be limited to maximum of 4 . Two simple filters selected are $h=\left[\begin{array}{ll}1 & 1\end{array}\right]$ or $\left[\begin{array}{lll}0.5 & 1 & 0.5\end{array}\right.$. First filter is a Haar smoothing filter with minimum number of taps. Second filter is a convolution of $\left[\begin{array}{lll}1 & 1\end{array}\right] \&\left[\begin{array}{ll}1 & 1\end{array}\right]$ with normalization. The main advantage gained through this choice is the scope for multiplication-less implementation.

\subsection{Implementation}

Block configuration for digital I-Q is indicated in figure 3 and implementation in figure 4. In this, sampled signal at $4 f_{0}$ is demodulated using $\cos (n \pi / 2) \& \sin (n \pi / 2)$. This sampled carrier takes the values $\{+1,0 \&-1\}$. For multiplication by $+1,2$ 's complement block passes on the input to its output. For -1 , it is reversed in polarity using 2's complement operation. Multiplication by zero is performed in association with Reg1 by resetting Reg1. Reg1, Reg2, Reg3 outputs form 3-tap filter [0.5 10.5 ] (register is a latch/delay element denoted as 'Reg' in this paper). For multiplication by 0.5 , adder $\sum_{1}$ inputs are given with a right shift. $\sum_{1}$ performs addition of first and the third sample. $\sum_{2}$ performs combined operation. This output is considered with suitable down sampling from 2 to 4 . Decimation operation is indicated as a down arrow with $M$ in the diagram. For the decimation factor $M$, PRI has to $i \tau M / 4$. where $i$ is an integer. With this condition for PRI an integer number of range bins are obtained and $M=4$ corresponds to one sample/pulse width. One sample per pulse width gives straddling loss and to avoid this loss, it is preferred to keep $M=3$.

For a selected $M$, hardware can be further simplified by optimizing the number of registers and adders. The main advantage of this scheme is the feasibility of configuring the whole hardware after ADC in Erasable Programmable Logic Devices (EPLD) or Field Programmable Gate Array (FPGA) without any multiplication operation. Hence, this type of scheme reduces hardware complexity.

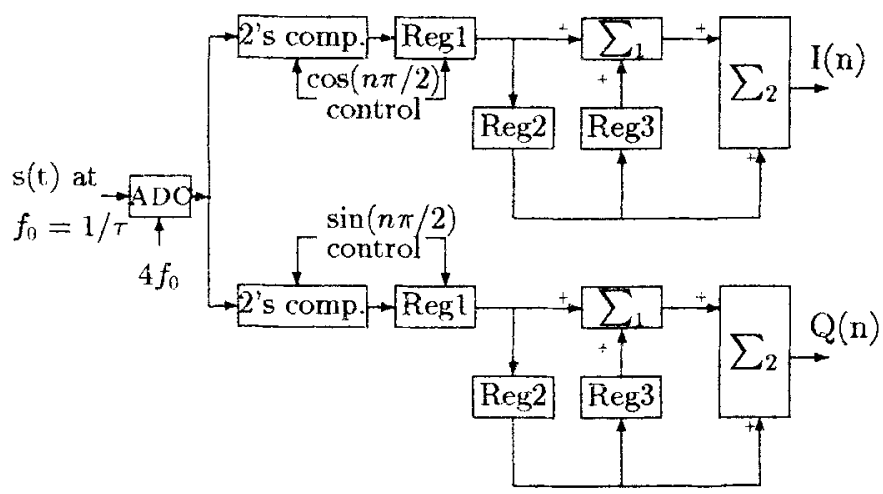

Figure 4. Digital I-Q implementation. 


\subsection{Imbalance correction}

As explained in the previous sections, digital I-Q requires frequency bin dependent correction. This has to be incorporated in the signal processors. However, this imbalance correction may not be required in the ground based and clutter Doppler locked pulse Doppler radars.

\subsection{Options}

(a) Sampled signal is demodulated using $\cos (n \pi / 2) \& \sin (n \pi / 2)$, passed through a low pass filter and decimated suitably.

(b) Sampled signal is demodulated as above and decimated by 2 or 4 in synchronism with the demodulating waveform (Liu et al 1989).

(c) Sample at highest possible frequency $n\left(4 f_{0}\right)$ to reduce phase imbalance, where ' $n$ ' is greater than or equal to 1 .

(d) For pulse compression waveforms like linear FM, the block LPF can be merged with the pulse compression filter in digital pulse compression schemes. This I-Q scheme is hardware intensive and so the possible options are to be considered depending on the application.

\section{Conclusions}

In this paper, different types of I-Q imbalance correction techniques are presented in time and frequency domains. These imbalance correction schemes are easy to implement in both hardware and software and avoid the use of matched I-Q detectors which are costly. It may be easier to implement these schemes in the frequency domain and the frequency domain data can be utilized for amplitude and phase imbalance estimation in the automated calibration procedure. This is an added advantage. The computations required in the above schemes are negligible compared to computation involved in DFT/FFT and other signal processing operations. For digital $\mathrm{I}-\mathrm{Q}$, it is preferred to go for frequency domain correction. Digital I-Q schemes are useful for radar and communication applications. These schemes with higher image rejection will be useful for air-borne Doppler radars. The optimum hardware can be configured for the desired parameters depending on specific application.

The authors would like to thank Prof. V U Reddy, for his invaluable suggestions. The authors would also like to thank Dr K V S S Prasada Rao and their colleagues for their support and encouragement. 


\section{References}

Brown J L 1979 On quadrature sampling of bandpass signals. IEEE Trans. Aerosp. Electron. Syst. AES-15: 366-371

Churchill F E, Ogar G W, Thompson B J 1981 The correction of I and Q errors in a coherent processor. IEEE Trans. Aerosp. Electron. Syst. AES-17: 131-137

Considine V 1983 Digital complex sampling. Electron. Lett. 19: 608-609

Frerking M E 1994 Digital signal processing in communication systems (New York: Van Nonstrand Reinhold) pp 113-151

Goldman S 1986 Understanding the limits of quadrature detection. Microwave \& $R F$ (Penton) Vol. 25, no. 13, pp 67-70\& 178

Levanon N 1988 Radar principles (New York: John Wiley)

Liu H, Ghafoor A, Stockman P H 1989 A new quadrature sampling and processing approach. IEEE Trans. Aerosp. Electron. Syst. AES-25: 733-747

Oppenheim A V, Schafer R W 1975 Digital signal processing (Englewood Cliffs, NJ: PrenticeHall)

Pierre J W, Fuhrmann D R 1995 Consideration in the autocalibration of quadrature receivers. Proc. Int. Conf. Acoust., Speech Signal Process., pp 1900-1903

Tsui J 1995 Digital techniques for wideband receivers (Norwood: Artech House) 